REVISTA
DA
FUNDARTE

\title{
LE JEU DE FICTION : ÉTUDE DE L’ACTUATION DE L’ENFANT EN SCÈNE Tiago Cruvinel
}

DOI: $10.19179 / 2319-0868.795$ 


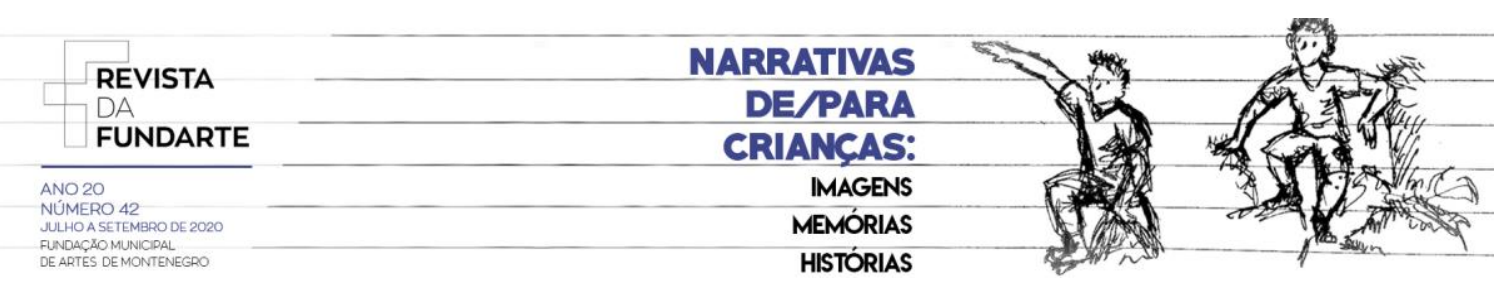

\title{
LE JEU DE FICTION : ÉTUDE DE L’ACTUATION DE L’ENFANT EN SCÈNE
}

Tiago Cruvine/ ${ }^{1}$

Resumo: O objetivo deste artigo é analisar a especificidade da brincadeira de ficção no contexto da atuação da criança em cena. A brincadeira de ficção pode ser entendida como uma forma de expressão corporal sofisticada, em que as crianças representam papeis sociais. O "faz de conta" aqui é percebido como a forma dramática primeira, de modo que essa informação e auxilia na compreensão do processo criativo da criança - via atuação/dramatização -, contribuindo com a preparação e a direção de criançasatores, dado que o "faz de conta" diz muito da cultura da própria criança, da capacidade natural que ela tem de atuar com seu corpo.

Palavras-chave: Criança; Brincadeira de ficção; Preparação; Cinema; Teatro.

\section{FICTIONAL PLAY: STUDY OF THE CHILD'S PERFORMANCE ON STAGE}

\begin{abstract}
The aim of this article is to analyse fictional playing specificity, once it can be understood as a sort of sophisticated body expression, in which kids play social roles. "Make-believe" is perceived here as the primary dramatic form, so that this information is essential and helps children's understanding of their creative processes - through acting/dramatization - contributing to children-actors preparation and direction, considering that "make-believe" tells much about kid's culture and their natural capacity of acting with their bodies.
\end{abstract}

Keywords: Child ; Fictional playing; Preparation.

\section{LE JEU DE FICTION : ÉTUDE DE L'ACTUATION DE L'ENFANT EN SCÈNE}

Résumé: L'objectif de cet article est d'analyser la spécificité du jeu de fiction de l'enfant. Celui-ci peut être défini comme une forme sophistiquée d'expression corporelle dans laquelle les enfants représentent des rôles sociaux. Le jeu de rôle de l'enfant est ici perçu comme la première forme d'expression dramatique. Cette information est fondamentale car elle aide à comprendre le processus créatif de l'enfant. Elle contribue aussi à la préparation et à la direction des enfants-acteurs. En effet, le jeu de rôle des enfants met en lumière la capacité naturelle de l'acteur à jouer avec son corps. II permet ainsi de mettre en perspective la spontanéité du comportement humain.

Mots clés : Enfant; Jeu de fiction; Préparation; Cinéma; Théâtre.

1 Ator e professor do Instituto Federal de Minas Gerais - IFMG - Campus Betim.

É doutor (2017) em Artes pela Universidade de Brasília - UnB e possui um pós-doutorado na Escola de

Belas Artes da Universidade Federal de Minas Gerais - UFMG. Autor do livro "Crianças em cena: Análise da atuação e de processos criativas com crianças-atores" (Editora CRV, 2015) e um dos

organizadores da série "Pedagogia das Artes Cênicas" (volumes 1, 2 e 3). Foi vice-coordenador do grupo de trabalho "Pedagogia das Artes Cênicas" da Associação Brasileira de Pesquisa e Pós-Graduação em

Artes Cênicas -

ABRACE. (2017-2018).

CRUVINEL, Tiago. Le jeu de fiction : étude de l'actuation de l'enfant en scène. Revista da FUNDARTE. Montenegro, p.01-12, ano 20, no 42, julho/setembro de 2020.

Disponível em: http://.seer.fundarte.rs.gov.br/index.php/RevistadaFundarte/index> 30 de setembro de 2020 


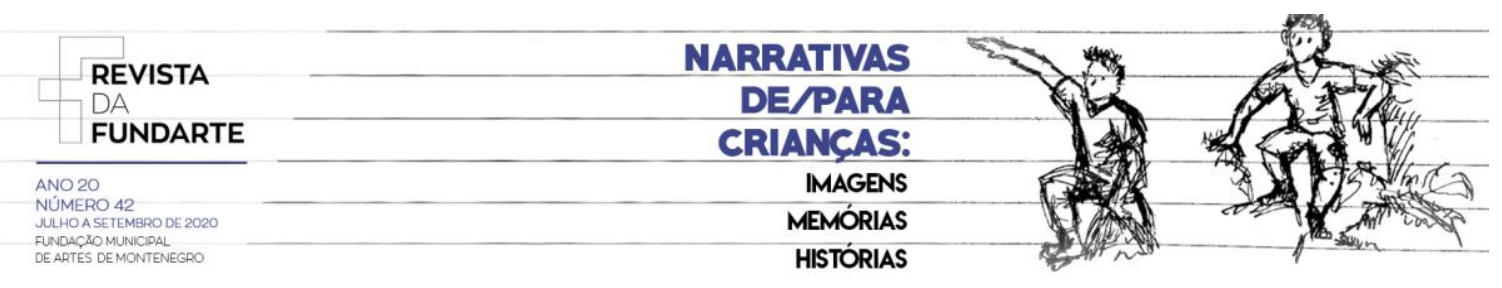

\section{Introduction}

Le sujet de mon étude théorique (CRUVINEL, 2017), concernant le processus de préparation de l'enfant-acteur dans le domaine cinématographique, a commencé par la compréhension du jeu fictionnel de l'enfant. Comment et pourquoi l'enfant joue le jeu de rôle? Se poser la question du «comment » et du «pourquoi » permet d'élargir les discussions à propos de l'approche scénique de l'enfant; dans un contexte professionnel comme scolaire.

Wallon (2007) a remarqué que dans les jeux de fiction les enfants reproduisent les impressions qu'ils viennent de vivre. Pour les enfants, la « règle » dans le jeu fictionsi l'on puit dire ainsi - serait l'imitation. Je reproduis, donc j'imite. Toujours d'après Wallon, cette imitation est produite par l'enfant car celui-ci - ayant des difficultés à dissocier réalité observable et fiction - n'arrête jamais d'alterner le recours aux deux. II observe parfois la fiction, parfois l'observation «II est toujours entrain de passer de l'une à l'autre. La réalité qu'il observe se mêle toujours à sa perception fictionnelle, tout comme cette dernière est saturée de son observation » (WALLON, 2007, p. 67).

Toujours du point de vue de l'auteur, l'enfant imitera ce qu'il a observé; ce qu'il y a de plus prestigieux pour lui; ce qui a atteint ses sentiments; ce qui touche généralement son affection. Cependant, l'enfant s'incarne dans les personnages qu'il interprète. II se retrouve totalement immergé dans son rôle, dans l'endroit dans lequel il s'imagine être (WALLON, 2007, p.67).

Ce qui s'observe ici dans le jeu de fiction, dans le « jeu de rôle », exige un minimum d'imitation, tant dans celle des objets que celle des personnages improvisés. De cette manière, quand un enfant construit un château de sable, joue au domino, coupe du papier, ou encore fait de la corde à sauter, - bien que ces activités soient des «expériences concrètes demandant un minimum d'éléments imaginatifs »

CRUVINEL, Tiago. Le jeu de fiction : étude de l'actuation de l'enfant en scène. Revista da FUNDARTE. Montenegro, p.01-12, ano 20, no 42, julho/setembro de 2020.

Disponível em: http://.seer.fundarte.rs.gov.br/index.php/RevistadaFundarte/index> 30 de setembro de 2020 


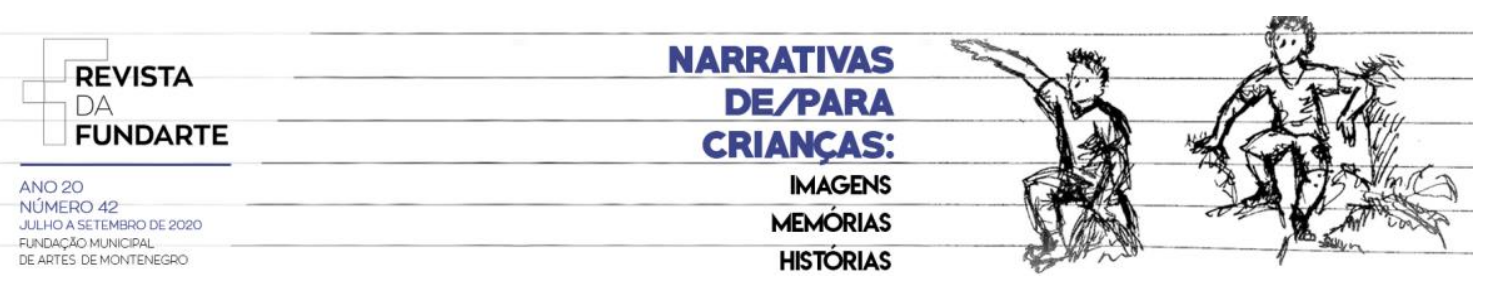

(BOMTEMPO, 1999, p.59), - elles ne relèvent pas de l'étude ici proposée si cet enfant n'a pas recours à une véritable imitation de l'objet ou du personnage.

Appuyant ce propos, Bomtempo donne l'exemple suivant : " un enfant de 4 ans dans une balançoire agit comme s'il était dans un avion et aurait besoin de changer les plans du vol parce qu'il a peu d'essence » (1999, p.60). Même si on peut se demander si l'enfant de quatre ans changerait ses plans de vol à cause d'un manque d'essence, pour Bomtempo, « il y a une différence entre ce type de jeu de rôle et le jeu d'un enfant qui simplement se balance le plus haut possible dans sa balançoire » (1999, p. 60). C'est le point clé de cet article. Dans le premier cas, l'enfant est en train d'imiter un rôle/personnage; dans le deuxième, il est seulement en train de chercher à vaincre le défi de pouvoir voler ou de dominer cette difficulté.

Selon l'historienne de l'art russe levguénia Petrova, citée par Vigotski (2009), «on peut analyser le jeu comme une forme dramatique première qui se différencie par une spécificité précieuse». Selon elle, le jeu réunit en une seule personne, «l'artiste, le spectateur, l'auteur de la pièce, le décorateur et le technicien » (PETROVA apud VIGOTSKI, 2009, p. 100). Le jeu aurait eu ainsi le caractère synthétique de tous ces éléments scéniques.

Vigotsky (2009), qui étudiait la création théâtrale à l'école, comprend que «le drame $^{2}$ serait directement lié au jeu, plus que n'importe quel autre type de création » (2009, p.99). Pour cette raison, le jeu serait plus synthétique, c'est-à-dire, «qu'il contiendrait des éléments créatifs des plus variés" (VIGOTSKI, 2009, p.99) (VIGOTSKI, 2009, p.99).

Dans le mot "jeu" du Lexique de pédagogie du théâtre (KOUDELA; ALMEIDA JUNIOR, 2015), élaboré par Igor de Almeida Silva, le jeu peut être compris comme

${ }^{2}$ Le mot drame, dans son étymologie, signifie action. Vinculé historiquement au théâtre, ce mot désigne "imitation par moyen d'action", c'est-à-dire, la dramatisation, "l'art de représenter (MOISÉS, Massoud. Diocnário de Termos Literários. São Paulo : Cultrix, 2004). 


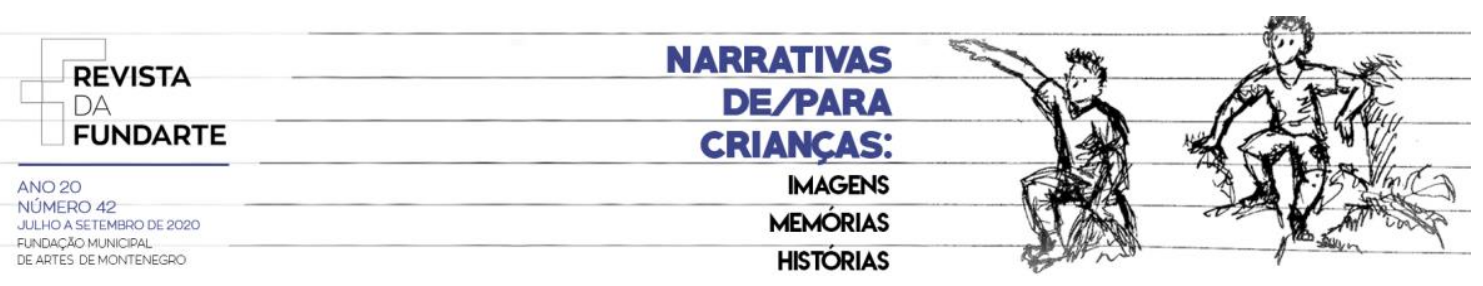

«ludique en action», autrement dit, «par intermédiaire de l'élément ludique, on peut penser le jouet ou le jeu en tant que création» (2015, p.23). Dans ce sens, «la mimésis du réel par l'enfant ne suppose pas nécessairement une copie, mais surtout, la création » (2015, p. 23). Le jeu est aussi vu, par l'écrivant du mot dans le Lexique, comme «l'espace-temps propre pour l'exercice de l'imagination ${ }^{3}$ » (KOUDELA; ALMEIDA JUNIOR, 2015, p. 24).

Alors, si le jeu peut être vu comme "ludique en action », et le "drame comme imitation d'une action », on perçoit que dans quelques jeux il existe la dramatisation (performance) au moment dont l'enfant fait son imitation du réel, bien plus si telle imitation n'est pas vue seulement comme une copie de quelque chose, mais comme une création. Dans cette perspective, il est identifié que l'enfant joue déjà comme un mécanisme de création et de renforcement de son imaginaire. Plus on joue le jeu de rôle, plus on devient habile pour gérer des situations qui partent des modèles imaginaires. Le jeu de fiction est un grand moment créé pour communiquer avec soimême, avec le monde extérieur et pour améliorer son imagination.

\section{À propos de l'imaginaire}

Je sais que l'utilisation du terme "imaginaire" peut gérer des ambigüités. Pour Maffesoli (2001), le problème du terme est qu'il a été utilisé comme opposition au réel, dans le sens que l'imaginaire sera toujours une fiction, sans consistance avec la réalité palpable (social, politique ou économique).

Toujours en relation avec les problématiques rencontrées par le terme imaginaire, on peut rajouter dans cette liste les perspectives de la psychanalyse et du constructivisme. Selon Sarmento (2003), ce que ces champs ont en commun, malgré

\footnotetext{
${ }^{3}$ Selon Vigotski (2009), la psychologie dénomme l'imagination, l'activité créatrice basée dans la capacité de combinaison de notre cerveau.
}

CRUVINEL, Tiago. Le jeu de fiction : étude de l'actuation de l'enfant en scène. Revista da FUNDARTE. Montenegro, p.01-12, ano 20, no 42, julho/setembro de 2020.

Disponível em: http://.seer.fundarte.rs.gov.br/index.php/RevistadaFundarte/index> 30 de setembro de 2020 


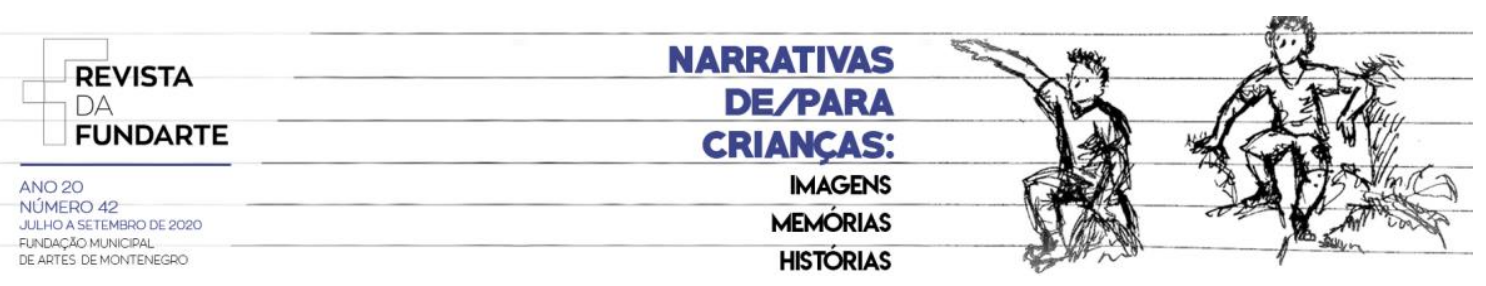

ses propres différences, a été concevoir l'imaginaire enfantin comme expression d'un déficit, c'est-à-dire, « les enfants imaginent le monde parce qu'ils manquent d'une pensé objective ou parce que ses liens rationnels avec la réalité sont imparfaitement formés » (SARMENTO, 2003, p. 2).

En révision avec les bases épistémologiques de ces champs, la Sociologie de l'enfance, par voie des études de Sarmento (2003) e des chercheurs dans le domaine, ont passée à contrarier cette conception du déficit, postulant que c'est dans l'ordre de la différence [entre le jeu de l'adulte et le jeu de l'enfant], et pas dans le déficit [immaturité enfantine], qui on parle d'un imaginaire enfantin. Ce qui existe c'est le principe de transposition imaginaire du réel, en étant l'imaginaire enfantin «inhérent au processus de formation et développement de la personnalité et de la rationalité de chaque enfant concret, mais cela se passe dans le contexte social et culturel qui fournit les conditions et les possibilités de ce processus » (SARMENTO, 2003, p. 3). On perçoit qu'il n'y a pas moyen de penser au réel et aux imaginaires comme opposés, en fait, ils se complémentent dans le processus de développement de l'enfant.

C'est pour cette raison que j'utilise le terme imaginaire, et aussi parce que « l'existence d'un imaginaire détermine l'existence de groupes d'images » (MAFFESOLI, 2001, p. 76). Encore selon Maffesoli : «II y a un imaginaire parisien qui gère une formation particulière de penser l'architecture, les jardins publics, la décoration des maisons [...] etc. L'imaginaire de Paris fait Paris être ce qu'elle est (MAFFESOLI, 2001, p. 76). Dans le cas de l'enfant, c'est à partir des modèles de son imaginaire, dans le jeu de rôle, que la pensée créée ou recrée des images et de nouvelles situations qui permet l'expansion de son imagination. Ici il n'importe pas si elles seront dans la sphère du palpable ou du qualifiable. Ce qui importe c'est que l'imaginaire modifie et transforme la réalité, c'est-à-dire, que s'agissant de l'enfant, je ne vois pas, comme je l'ai déjà dit, l'imaginaire comme opposition à la réalité; il se rajoute à la réalité pour le développement des moyens d'être de l'enfant.

CRUVINEL, Tiago. Le jeu de fiction : étude de l'actuation de l'enfant en scène. Revista da FUNDARTE. Montenegro, p.01-12, ano 20, oㅡ 42, julho/setembro de 2020.

Disponível em: http://.seer.fundarte.rs.gov.br/index.php/RevistadaFundarte/index> 30 de setembro de 2020 


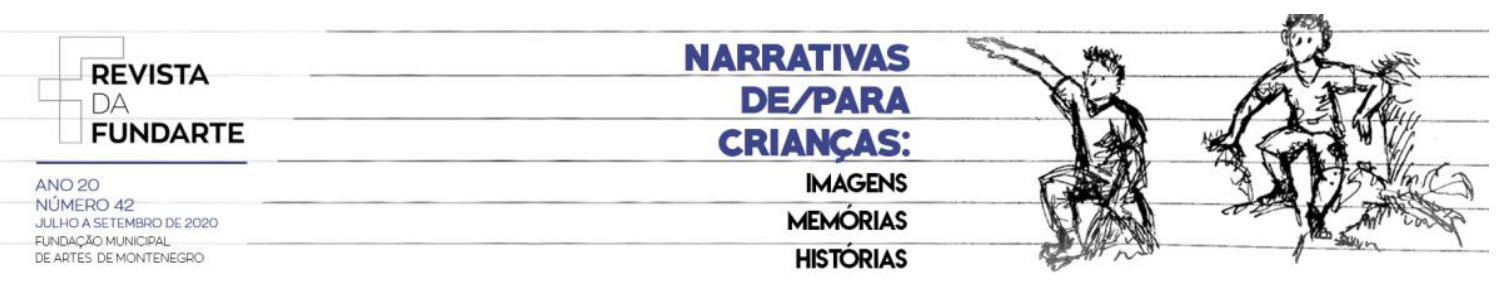

Le degré maximum de la performance, dans le jeu d'enfant, dans la modernité connue comme "jeu de rôle ", se passe quand l'activité gère plaisir et l'action est proposé par l'enfant lui-même; quand il y a la présence des dialogues réels ou imaginaires; quand il y a l'élaboration de rôles - en quoi il se met à la place d'un objet réel ou en situation imaginaire (comme un agent policier ou un arbre) -; et quand le regard de cette autre (personne ou objet) est défini, en lui dirigeant à comprendre son action, parce que l'objectif n'est pas parler tout seul, même s'il faut parler avec une poupée.

Voici un exemple d'un enfant performant dans son jeu fictionnel, à partir du découpage proposé dans cet article:

Une fille, qui a enfoui ses pieds dans la terre et qui s'est tenue immobile avec les bras collés au corps, a dit: "Je suis un arbre. Tu ne vois pas ? Je grandis. Regarde les branches, les petites feuilles". Ses mains commencent à se lever doucement, les petits doigts s'ouvrent. "Tu ne vois pas comment le vent me secoue? " - Et "l'arbre" commence à s'incliner et à trépider avec les petites feuilles-doigts. (PETROVA apud VIGOTSKI, 2009, p. 99).

Toujours dans l'essai d'expliquer comment l'enfant joue le « jeu de rôle », on voit que la performance dans le jeu est dans la situation imaginaire que l'enfant crée à partir du dialogue avec l'autre, pouvant celui-là être une personne ou un objet, comme une poupée, en tenant compte que beaucoup d'enfants tiennent un dialogue avec ses jouets. La situation - enterrer ses pieds dans la terre rester immobile - présente les caractéristiques du «jeu de rôle », c'est-à-dire, par moyens de "faire comme si », l'enfant si positionne à la place d'un objet réel, ou d'une situation imaginaire, par moyen d'imitation. "Le jeu de rôle est le jeu d'enfant avec son imaginaire » (KOUDELA; ALMEIDA JUNIOR, 2015, p. 75).

« Je suis un arbre. Tu ne vois pas ? - dans cet exemple, l'enfant crée son rôle, en confirmant son action antérieure de se planter. « Regarde les branches, les petites

CRUVINEL, Tiago. Le jeu de fiction : étude de l'actuation de l'enfant en scène. Revista da FUNDARTE. Montenegro, p.01-12, ano 20, no 42, julho/setembro de 2020.

Disponível em: http://.seer.fundarte.rs.gov.br/index.php/RevistadaFundarte/index> 30 de setembro de 2020 


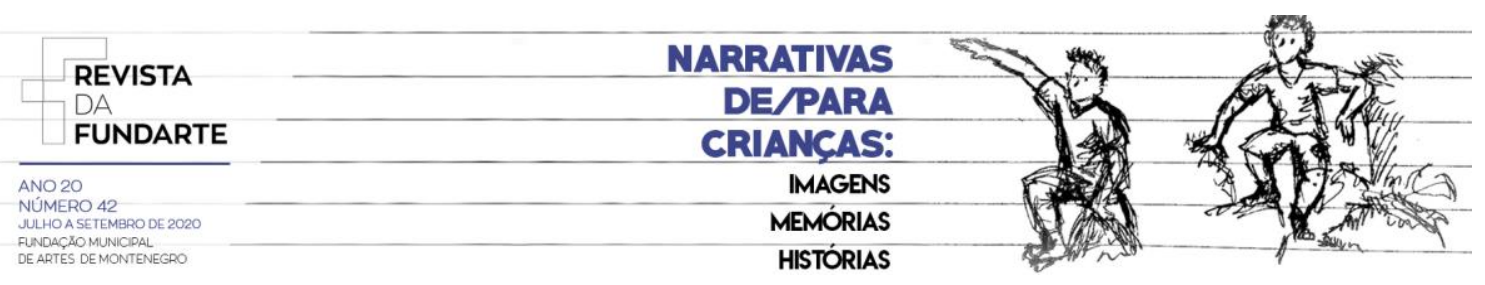

feuilles » - dans ce dialogue, l'enfant exige l'expansion de l'imagination de qui regarde l'action, en dirigeant le regard de qui voit, en dirigeant tel regard. L'inclination de l'arbre et la floraison des petites feuilles ne sont pas seulement un détail dans le jeu, mais une conclusion de l'action : « Je suis un arbre [rôle/personnage] et « Je grandis » [action].

Laurence Rameu (2014), pédiatre et auteure du livre Pourquoi les bébés jouent? (2011), après analyser le pourquoi les enfants jouent, elle comprend que le jeu n'a aucun objectif à priori, juste le plaisir qu'elle sent en pratique. C'est-à-dire, personne ne joue pour obligation. Pour l'auteure, à partir du plaisir dans le jeu, l'enfant ira explorer, expérimenter et apprendre, pas seulement le fonctionnement du monde psychique et social, tel qu'il est, mais avec d'autres mondes possibles è partir de son imaginaire. De la même manière, Rameau (2014) comprend que le jeu est utilisé pour le développement : contrairement à ce que beaucoup pensent, l'enfant ne joue pas pour apprendre, mais c'est parce qu'il joue qu'il apprend (RAMEAU, 2014). En d'autres termes, l'obligation à l'opposé de l'expérimentation du langage cinématographique ou théâtral, ce qui contribues-en même, comme nous le verrons, dans le développement humain. De plus, c'est par plaisir, le désir, par exemple que les enfants apprendront sur l'expérience de jouer (un jeu ou un rôle) pour réussir à développer un rôle/personnage particulier dans un film.

En revenant à la question sur la situation imaginaire, l'arbre, dans l'exemple cité, est créé à partir de l'observation en soi, de l'observation de la nature, comme si le jeu fictionnel avait été basé dans un point de la réalité. C'est l'observation qui gère les modèles imaginaires de l'enfant.

La création des modèles imaginaires basés dans l'observation de la réalité ${ }^{4}$ est un aspect relevant et qui dialogue aussi avec la notion de jeu cinématographique. J'amène, aussi, un questionnement abordé dans ma thèse de doctorat (CRUVINEL,

4 Réalité aussi dans le sens de ce qui est au tour de l'enfant - pour englober tous les types de stimulations, soient visuelles, comme dessins animés, ou audiovisuels, comme musiques, entre autres.

CRUVINEL, Tiago. Le jeu de fiction : étude de l'actuation de l'enfant en scène. Revista da FUNDARTE. Montenegro, p.01-12, ano 20, no 42, julho/setembro de 2020.

Disponível em: http://.seer.fundarte.rs.gov.br/index.php/RevistadaFundarte/index> 30 de setembro de 2020 


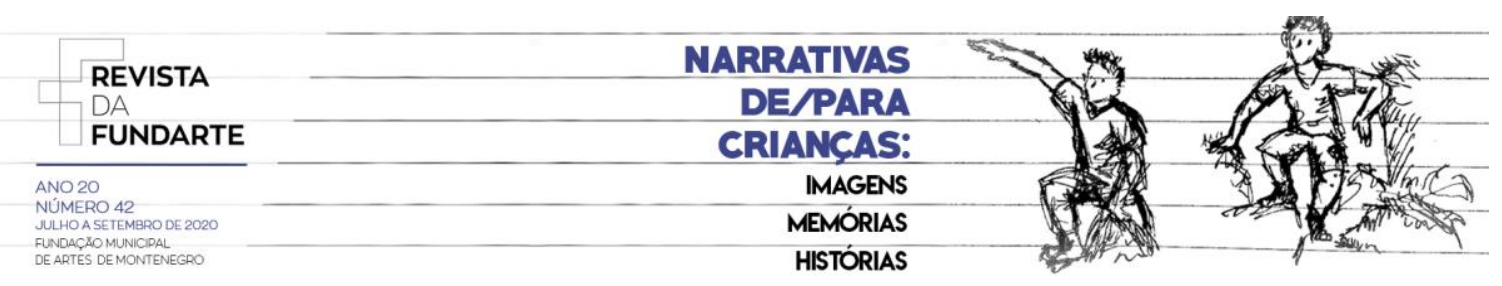

2017), pour faire la connexion avec le principe de jeu présenté dans cet article : comme un enfant de quatre va jouer un personnage s'il n'a pas la maitrise nécessaire pour développer une telle activité ?

\section{L'enfant-acteur dans le cinéma: «le jeu de rôle » dans son territoire}

Il est très courant que la production d'un filme réalise un casting en essayant le maximum de contexte réel des personnages-enfants désignés dans le scénario, cela est clair quand on pense dans une esthétique réaliste. Par exemple, selon la critique de cinéma Zoé Protat (2011), le réalisateur de cinéma français Robert Bresson [19011999], du mouvement minimaliste, a choisi ses acteurs pour ce qu'ils sont en réalité.

Un autre exemple: la production du filme Slumdog Millionnaire (2009), de Danny Boyle, a réalisé des tests avec 1.500 enfants de bidons-villes en Inde, en comprenant que ces enfants s'approchent plus, en termes de réalisme, des personnages-enfants, mais aussi parce que ces enfants étaient déjà immergés dans une culture déterminée et ont pu les observer, les analyser et les critiquer $^{5}$, en étant plus facile créer, dans la majorité de cas, un modèle imaginaire, un personnage, à partir de la réalité observée et déjà partagée entre le personnage et l'enfant-acteur. Ce personnage peut être expliqué dans le fragment dont Rubina Ali - l'enfant-actrice qui a été choisie pour jouer le personnage Latika - décrit quelques-unes des scènes et le processus du filmage. Dans l'occasion, elle a dit :

\footnotetext{
${ }^{5}$ Un bon exemple de la capacité d'analyser de manière critique le contexte dans lequel on vit ça peut être vu dans le livre de Rubina Ali (2010), un enfant qui a joué dans le film ... en collaboration avec Anne Anne Berthold et la participation de Divya Dugar. Rubina, à ses 9 ans, a écrit le livre pour raconter son histoire de vie avant, pendant et après le film. Le livre est intéressant parce qu'il présente une vision en temps réel, critique et poétique à propos des bidonvilles de l'Inde, à partir du regard d'un enfant qui désire devenir une actrice professionnelle.
}

CRUVINEL, Tiago. Le jeu de fiction : étude de l'actuation de l'enfant en scène. Revista da FUNDARTE. Montenegro, p.01-12, ano 20, no 42, julho/setembro de 2020.

Disponível em: http://.seer.fundarte.rs.gov.br/index.php/RevistadaFundarte/index> 30 de setembro de 2020 


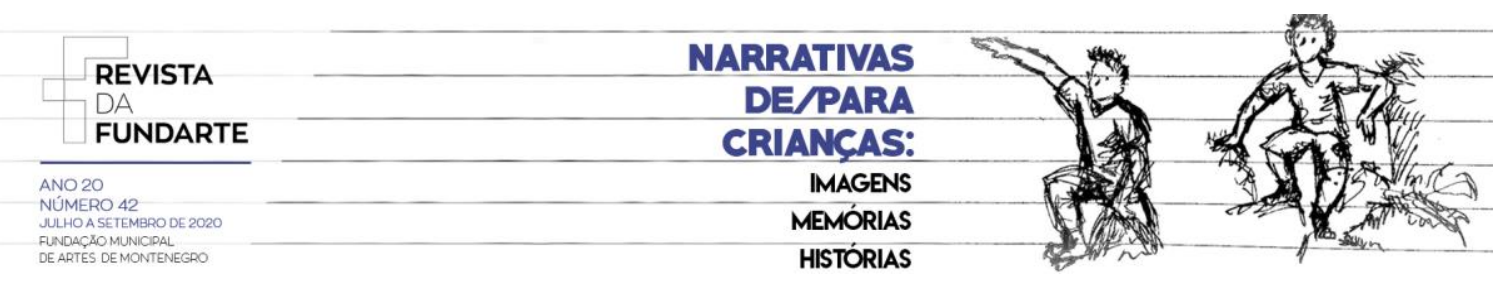

Ayush, Azhar6, et moi avions beaucoup de joie pour filmer les extérieurs, en Dharavi ${ }^{7}$. Ce sont des endroits très grands, avec tant de gens et tant de magasins dans tous les sens, qu'il est facile se perdre. Quand on nous a demandé pour courir dans les ruelles étroites de Dharavi, ce sont les caméras qui ont eu de la difficulté à nous suivre! On était habité à sauter directement dans une flaque d'eau sale avec les pieds nus, à courir entre les vaches et les personnes dans les passages pas suffisamment larges pour quelqu'un. C'était comme si on jouait au cache-cache dans le bidonville, sauf qu'il a fallu refaire le même parcours à un nombre incalculable jusqu'à que l'oncle Danny soit satisfait avec le plan-séquence (ALI, 2010, p. 51).

Peut-être qu'un autre enfant, d'un autre pays, même si de la même culture, mais qui n'était pas en train de vivre la réalité des bidonvilles en Inde, aurait eu une plus grande difficulté en réaliser ces scènes décrites par Rubina Ali. Premièrement, par l'étrangeté de la région, des costumes, de la pauvreté ou d'autres aspects particulaires de chacun; selon, pourquoi le personnage et l'enfant-acteur n'ont pas vécu la même réalité. En d'autres termes, le modèle pour la création de l'imaginaire, nécessaire pour l'élaboration du personnage, ne serait pas le même de celui déjà partagé entre l'enfantacteur et le personnage. Dans ma thèse de doctorat (CRUVINEL, 2017), je remets en question les types de jeu que chaque film exige de l'enfant, et peut-être que cette logique ne convient pas à tous les contextes, mais j'affirme qu'il s'agit d'une pratique très courante dans le cinéma. Des enfants-acteurs sont recherchés pour réaliser le casting dans la propre réalité proposée par le scénario du film, comme si le cinéma réaliste recherchait toujours le « cache-cache dans son territoire », je dirais, pour faire un lien avec le témoignage de Rubina Ali, et par la suite, transposer à l'écran, des situations plus proches du contexte réaliste traité par le scénario.

\section{Considérations finales}

\footnotetext{
${ }^{6}$ Ayush Mahesh Khedekar, Azharuddin Mohammed Ismaïl et Rubina Ali jouent les trois rôles principaux, quand ils étaient enfants.

7 Dharavi est un bidonville situé au centre de Mumbai. Rubina Ali, qui nous fournit ce témoignage, vivait dans un autre bidonville le Garib Nagar, connu comme "La ville des pauvres", un autre arrondissement de Mumbai.
}

CRUVINEL, Tiago. Le jeu de fiction : étude de l'actuation de l'enfant en scène. Revista da FUNDARTE. Montenegro, p.01-12, ano 20, no 42, julho/setembro de 2020.

Disponível em: http://.seer.fundarte.rs.gov.br/index.php/RevistadaFundarte/index> 30 de setembro de 2020 


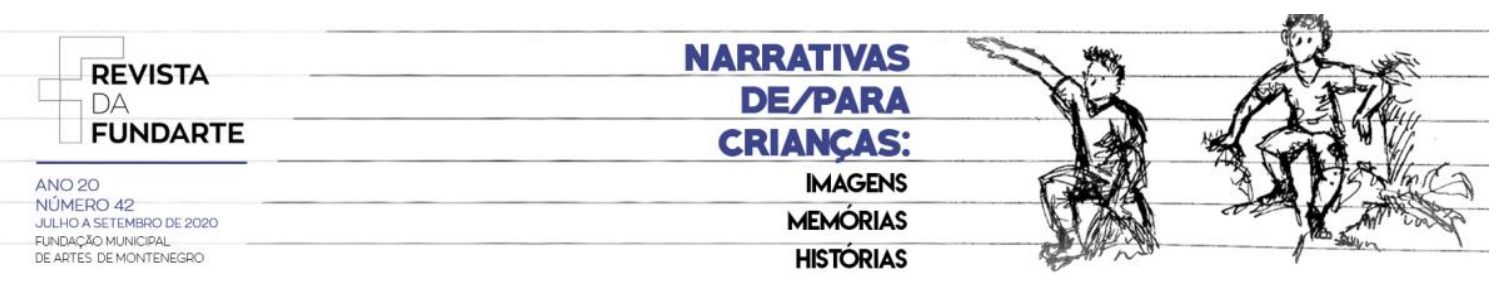

Les différences que je présente dans cet article sont pertinentes pour comprendre quels sont les objectifs du "jeu de rôle ». En bref, l'enfant joue pour luimême en dialoguant avec l'autre (objets ou personnes), dans le jeu fictionnel, à partir de son propre imaginaire, des modèles ressortis de sa réalité. Au moment d'écrire le scénario, on voit qu'il y a une orientation de la part de l'adulte, en créant une relation de jeu, et non de plaisanterie, avec l'enfant-acteur. C'est l'auteur qui crée la première règle du jeu.

De cette manière, comprendre le jeu fictionnel, en tant que première forme dramatique, nous aide à mieux comprendre le processus de création et de direction des enfants-acteurs. Ainsi, revisiter les concepts nous permet de mieux élaborer les méthodes d'actuation et de mieux comprendre le mode d'être (quelqu'un) et d'être (quelque part) de l'enfant dans le monde avec son corps.

\section{Références:}

ALI, Rubina. O sonho de Rubina. Escrito em colaboração com Anne Berthod e com a participação de Divya Dugar. São Paulo: Editora Planeta do Brasil, 2010.

BOMTEMPO, Edda. A brincadeira de faz de conta: lugar do simbolismo, da representação, do imaginário. In: KISHIMOTO, Tizuko (Org.) Jogo, brinquedo, brincadeira e educação. São Paulo: Cortez,1999. p. 57-72.

CRUVINEL, Tiago de Brito. A criança-ator no cinema: jogando na sétima arte. 2017. 195 f., il. Tese (Doutorado em Arte) — Universidade de Brasília, Brasília, 2017.

CRUVINEL, Tiago. Criança em cena: análise da atuação e de processos criativos com crianças-atores. Curitiba: Editora CRV, 2015.

KOUDELA, Ingrid; ALMEIDA JÚNIOR, José Simões (Org.) Léxico de pedagogia do teatro. São Paulo: Perspectiva; SP Escola de Teatro, 2015.

CRUVINEL, Tiago. Le jeu de fiction : étude de l'actuation de l'enfant en scène. Revista da FUNDARTE. Montenegro, p.01-12, ano 20, no 42, julho/setembro de 2020.

Disponível em: http://.seer.fundarte.rs.gov.br/index.php/RevistadaFundarte/index> 30 de setembro de 2020 


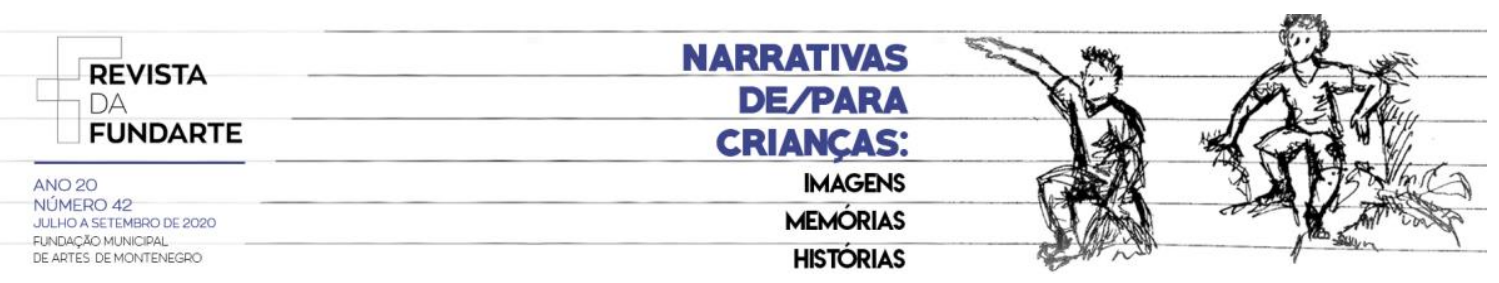

LES ÉPHÉMĖRES. Filmado por Bernard Zitzermann, Mise en scène de Ariane Mnouchkine. França: Le Théâtre du Soleil, Bel Air Media, ARTE France, Centre National de la Cinématographie, 2009. 1 filme (5h47min), sonoro, legenda, color. 16/9.

MAFFESOLI, Michel. O imaginário é uma realidade (entrevista). In: Revista Famecos: mídia, cultura e tecnologia. Porto Alegre, v. 1, n. 15, p. 74-82, ago. 2001.

MOISÉS, Massaud. Dicionário de termos literários. São Paulo: Cultrix, 2004.

PONETTE. Direção de Jacques Doillon, 1996. França: FOX LORBER: OBRAS PRIAS [Edição especial de colecionador]. 1 filme (97 min.), sonoro, color., NTSC.

PROTAT, Zoé. La direction d'acteur - jouer de confiance. Ciné-Bulles, v. 29, n. 1, p. 38-41, 2011.

QUEM QUER SER UM MILIONÁRIO? Direção de Danny Boyle. 2009. Reino Unido: Warner Bros. Pictures. 1 filme (120 minutos), sonoro, color., NTSC.

RAMEAU, Laurence. Pourquoi les enfants jouent-ils?. In: Psychologie de l'enfant: état des lieux. Sciences Humaines, France, n. 259, p. 44-45, Mai., 2014.

SARMENTO, Manuel. Imaginário e culturas da infância. Cadernos de Educação (Pelotas), v. 12, p. 1-18, 2003. Disponível em: $<$ http://www.titosena.faed.udesc.br/Arquivos/Artigos_infancia/Cultura\%20na\%20Infancia .pdf>. Acesso em 7 jun. 2017.

VIGOTSKI, Lev. Imaginação e criação na infância. Tradução de Zoia Prestes. São Paulo: Ática, 2009.

WALLON, Henri. A evolução psicológica da criança. Tradução de Claudia Berliner. São Paulo: Martins Fontes, 2007.

CRUVINEL, Tiago. Le jeu de fiction : étude de l'actuation de l'enfant en scène. Revista da FUNDARTE. Montenegro, p.01-12, ano 20, no 42, julho/setembro de 2020.

Disponível em: http://.seer.fundarte.rs.gov.br/index.php/RevistadaFundarte/index> 30 de setembro de 2020 